\title{
Brainwave Classification of Task Performed by Stroke Patients using ANN
}

\author{
S.K. Narudin*, N.H.M. Nasir and N. Fuad \\ Universiti Tun Hussein Onn Malaysia, Malaysia \\ he180051@siswa.uthm.edu.my; hamizahn@uthm.edu.my; norfaiza@uthm.edu.my \\ *Correspondence: he180051@siswa.uthm.edu.my
}

\begin{abstract}
Received: 17th October 2020; Accepted: 18 ${ }^{\text {th }}$ December 2020; Published: 20 ${ }^{\text {th }}$ March 2021
Abstract: In this research, 14 stroke patient's brainwave activity with open eyes (OE) and close eyes (CE) sessions are used. This work aims to study and classify 2 activities that validate our data acquisition. The data set of each subject is used to classify the state of the subject during electroencephalogram (EEG) recording. For the classification model, the input signals are alpha, beta, theta, and delta bands. The classification algorithm used in this work is the Artificial Neural Network (ANN). The accuracy value will be obtained from each subject. There are substancial differences between the EEG signals of each patient and hence affecting the accuracy value of the subject. The results obtained from our experiment proved that ANN can be used to classify the state of the subject during data recording.
\end{abstract}

Keywords: EEG; Stroke; Classification; ANN

\section{Introduction}

Stroke has been one of the primary causes of worldwide disabilities. Stroke-related motor function deficits lead to poor overall life quality [1]. Brain-Computer Interface (BCI) provides a communications medium and interaction with a system directly from the brain in an external environment, without any motor pathway being involved. The basis for regulating BCI systems is neurological phenomena which are special features of brain activity occurring in the brain signals. Various approaches to capturing brain signals and analyzing neurological phenomena were employed. One of the methods is EEG which records electrical activity along the scalp surface [2]. An EEG-based BCI detects a patient's neuronal signal as an input, enabling users to monitor their brain function effectively.

The stroke classification during stroke treatment and recovery has many implications. It works as a reference for prediction, recommendation of treatment, and secondary stroke prevention approaches [3],[4],[5]. A classification is the method of obtaining a number of models that define and separate data classes for the use of unknown class label prediction [6].

The ANN is among the easiest methods for classification using a machine learning algorithm. ANN have been extensively used for the system of non-linear modelling [3], applications of medical [7], and pattern recognition [8]. ANN is composed of layers of input, hidden, and output. The weights which connect the layers indirectly form the system. The input and the output connection determine network behavior. In this work, ANN will be used to classify 2 types of the task performed by 14 stroke subjects. The classification performance in each subject will be observed by obtaining accuracy value from each subject. Although there are many comprehensive works dedicated to brainwave classification of stroke patients, little work has been carried out to determine the classification of each 
stroke subjects by task using scaled conjugate gradient backpropagation.

\section{Literature Review}

Aowlad Hossain et al. [9] in 2015 had conducted research on the EEG signals classification of movement of left and right-hand. Within this study, the key goal is to classify EEG signal of left and right-hand movement based on wavelet transform and neural network approach. Discrete wavelet transforms (DWT) has been used to split the beta band from the EEG signal. The performance of a PNN is evaluated in order to discover a better classifier of the EEG signals and compared with the classical backpropagation neural network. From the research, PNN with 99.1\% accuracy shows a better classification rate than BP with $88.9 \%$ accuracy.

Thiyam et al. [10] in 2016 had proposed analysis for the classification of multiclass motor imagery movements. In their experiments, the classifiers were trained by the extracted features and the testing features were used to attained classification performance. The classification performance analysis of Support Vector Machine (SVM) and Linear Discriminant Analysis (LDA) are analyzed using two pre-processing algorithms which are a multiclass common spatial pattern (mCSP) and thin ICA-CSP algorithms using the multiclass motor imagery movements EEG signals. The accuracy of LDA and SVM using mCSP techniques are $62 \%$ and $60 \%$ respectively. While by using thin ICA-CSP, the accuracies are $64 \%$ and $63 \%$ for LDA and SVM respectively. In overall, the LDA performs better than SVM for discrimination of multiclass movement for BCI competition IV dataset $2 a$.

A particle swarm optimization algorithm was proposed by Ma et al. [11] in 2016 to increase the classification accuracy of SVM by optimizing the selection of both kernel and penalty parameters. He conducted experiments by using 2 different data sets: 2005 Data Iva and 2008 Dataset 1. Both data sets were used for the classification of PSO-SVM and the classical method. The classical methods used are decision tree, back propagation., k-NN, LDA, and SVM. The result reveals that the PSO-SVM shows the highest percentage of accuracy.

Also, a method of using SVM, k-NN, and BP to classify EEG signals for different brain control machines had been studied by Islam et al. [12] in 2017. EEG datasets that are built with various cognitive tasks such as left, right, back and front imaginary movement with eyes open were presented. The proposed work obtained the highest accuracy by $95.21 \%$ for SVM.

Suwannarat et al. in [13] 2018 had experimented to compare the EEG data of upper limb movement using motor imagery. Both right- hand and left-hand motor imagery tasks which were open/close, bracket flexion and extension, and forearm pronation/supination are used. The experiment was attended by eleven subjects. Every MI task consisted of 8 sessions lasting for 4 weeks. Feature extraction using the common space pattern (CSP) algorithm has been used for classification. Conventional CSP (WB) and one with an increasing features number attained by EEG data filtering into five bands (FB) were implemented. LDA and SVM classification were carried out. The accuracy of FB is significantly higher than the WB. The accuracy of classification of the wrist flexion/extension task in all subjects was higher than that of the classification of hand opening/closing. In most subjects, the classification of the forearm pronation/supination task achieved greater accuracy than the classification of the manual open/close hand task but less accuracy than the classification of the wrist flexion/extension task in all subjects. The accuracy classification of the wrist flexure/extension and the task of forearm pronation/suppression was lower than the accuracy of the tasks of hand movement and wrist motion.

All the previous works that had been discussed are summarized in Table 1.

Table 1. Summary of previous works

\begin{tabular}{|l|l|l|l|l|l|l|l|l|l|}
\hline Year & Previous Works & LDA & SVM & k-NN & BP & PNN & $\begin{array}{l}\text { Decision } \\
\text { Tree }\end{array}$ & others \\
\hline 2015 & \multicolumn{2}{|l|}{ Aowlad Hossain et al. } & - & - & - & $88.9 \%$ & $99.1 \%$ & - & - \\
\hline 2016 & Thiyam et al. & mCSP & $62 \%$ & $60 \%$ & - & - & & - & - \\
\cline { 3 - 10 } & & $\begin{array}{l}\text { thin ICA- } \\
\text { CSP }\end{array}$ & $64 \%$ & $63 \%$ & - & - & & - & - \\
\hline
\end{tabular}




\begin{tabular}{|c|c|c|c|c|c|c|c|c|}
\hline \multirow[t]{2}{*}{2016} & \multirow[t]{2}{*}{ MA et al. } & $\begin{array}{l}2005 \text { Data } \\
\text { Iva }\end{array}$ & $85.4 \%$ & $96.4 \%$ & $92.5 \%$ & $84.2 \%$ & $79.8 \%$ & $\begin{array}{l}97.0 \% \\
\text { (PSO- } \\
\text { SVM) }\end{array}$ \\
\hline & & $\begin{array}{l}2008 \\
\text { Dataset } 1\end{array}$ & $90.8 \%$ & $86.8 \%$ & $87.1 \%$ & $85.4 \%$ & $84.5 \%$ & $\begin{array}{l}89.7 \% \\
\text { (PSO- } \\
\text { SVM) }\end{array}$ \\
\hline 2017 & \multicolumn{2}{|l|}{ Islam et al. } & - & $95.21 \%$ & $90.88 \%$ & $94.31 \%$ & - & - \\
\hline \multirow[t]{3}{*}{2018} & \multirow[t]{3}{*}{ Suwannarat et al. } & $\begin{array}{l}\text { Opening/ } \\
\text { closing }\end{array}$ & $64.07 \%$ & $64.02 \%$ & & & & $\begin{array}{l}61.9 \% \\
(\mathrm{WB}), \\
66.19 \% \\
(\mathrm{FB})\end{array}$ \\
\hline & & $\begin{array}{l}\text { Flexion/ } \\
\text { tension }\end{array}$ & $68.79 \%$ & $68.71 \%$ & & & & $\begin{array}{l}66.4 .9 \% \\
(\mathrm{WB}), \\
71.10 \% \\
(\mathrm{FB})\end{array}$ \\
\hline & & $\begin{array}{l}\text { Pronation/ } \\
\text { supination }\end{array}$ & $69.10 \%$ & $68.97 \%$ & & & & $\begin{array}{l}66.38 \% \\
\text { (WB), } \\
71.69 \% \\
\text { (FB) }\end{array}$ \\
\hline
\end{tabular}

\section{Methodology}

A BCI is a synthetics intelligent system able to understand a specific collection of trends in brain signals namely, acquisition of signal, pre-processing, extraction of feature, classification, and interface for the practical application of the system [14]. Figure 1 shows the flow chart of the methodology used in this paper. This consists of a sequence of EEG data compilation, data pre-processing, extraction of feature, and classification.

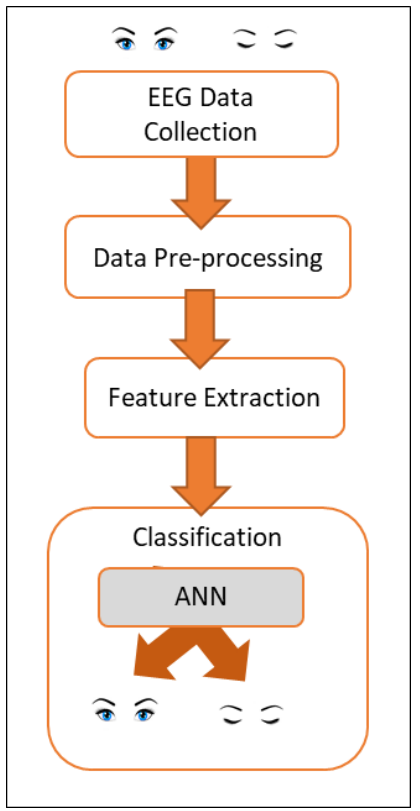

Figure 1. Flow diagram of the methodology

The data were collected at the National Stroke Association of Malaysia (NASAM), Selangor, Malaysia. The participants took part in the data collection voluntarily. They are 14 stroke patients aged between 40-60 years old. Initially, the stroke groups were graded according to analytic and examination by trained NASAM physiotherapist which are advanced and early group. However, both characteristics are neglected for this experiment since the experiment requires data from stroke patients at any level.

Throughout the experiments, the subjects were instructed to be seated comfortably in front of a computer screen to perform open eyes and close eyes task respectively. Data will go through signal preprocessing, feature extraction, and classification phase. If the information contains many artefacts, then pre-processing methods may be required that are more complicated in removing or reducing 
any unwanted artefact impacts. It is also important to minimize psychological and environmental distinctions [15]. Scaled conjugate gradient backpropagation. is applied in the classification method to classify the task performed by the subject (open/close eyes) according to their brainwave pattern. Figure 2 shows an example of a subject goes through the EEG recording during close eyes session.

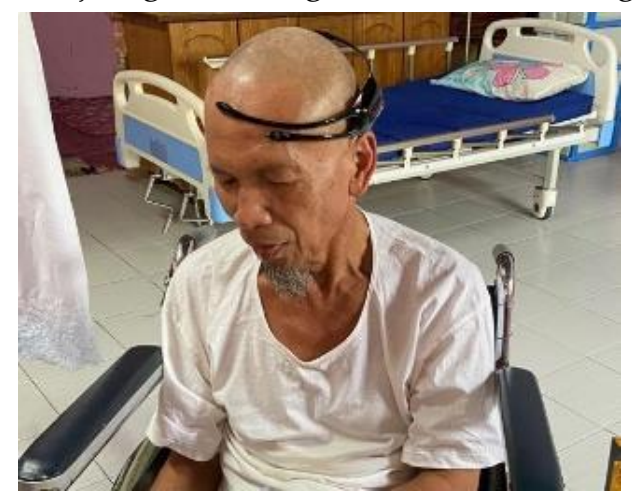

Figure 2. A subject goes through close eye EEG recording

\subsection{EEG Signal Analysis}

EEG data were filtered to eliminate the artefact for example the the blink of the eye, baseline noise and interference of power line. By removing the sampled data with an amplitude above $100 \mu \mathrm{V}$ and voltage characteristics below $-100 \mu \mathrm{V}$, the non-EEG signal was removed. The signals produced are then filtered into delta band, theta band, alpha band, and beta band. The EEG signal of OE and CE from 14 stroke individuals is displayed in Figure 4.

\subsection{Neural Network Model during the Open-Close Eyes Task}

The ANN model consists of 4 input nodes which are the alpha, beta, delta, and theta power band of each subject while the output nodes are the tasks performed by the subjects. In this case, the tasks are open eyes and close eyes. 10000 sample data for each subject are used. The features have been randomly distributed the 10000 samples for training, validation, and test dataset with percentage ratio 7 0:15:15. A training dataset is introduced during training to the network and the network is modified due to its error while the validation dataset is used to measure network generalization, and to avoid training when generalizations stop improving. However, a testing dataset does not affect training and so provides an independent measure of network performance during and after training. Validation dataset is expected to avoid overfitting of the network when testing data has been used to assess the performance of the classification.

A two-layer feed-forward network, with SoftMax output neurons and sigmoid hidden neurons, can randomly classify victory, and provide the hidden layer with sufficient neurons. Scaled conjugate gradient backpropagation will be used to trained the network. The adaptation of the weights between output $(\mathrm{k})$ and hidden (j) layers are defined as follows:

$W_{k j}(t+1)=W_{k j}(t)+\triangle W_{k j}(t+1)$

Where

$$
\triangle W_{k j}(t+1)=\eta \delta_{k} O_{j} \quad \triangle \alpha W_{k j}(t)
$$

Where $t$ is the iteration number and $\delta_{k}$ is the error signal between the hidden and output layers:

$\delta_{k}=O_{k}\left(1-O_{k}\right)\left(t_{k}-O_{k}\right)$

Adaptation between hidden (j) and input (i) layers:

$\triangle W_{j i}(t+1)=\eta \delta_{j} O_{i} \triangle \alpha W_{j i}(t)$

The new weight is thus:

$W_{j i}(t+1)=W_{j i}(t)+\triangle W_{j i}(t+1)$

And the error signal through layer $\mathrm{j}$ is:

$\delta_{i}=O_{j}\left(1-O_{j}\right) \sum_{k} \delta_{k} W_{k j}$ 
The model has been improved to gain the momentum constant, hidden neurons number, and learning rate that can give the highest accuracies in training and validation dataset [16]. The final mode is selected based on the best accuracy with the least error. Figure 3 illustrates the ANN model.

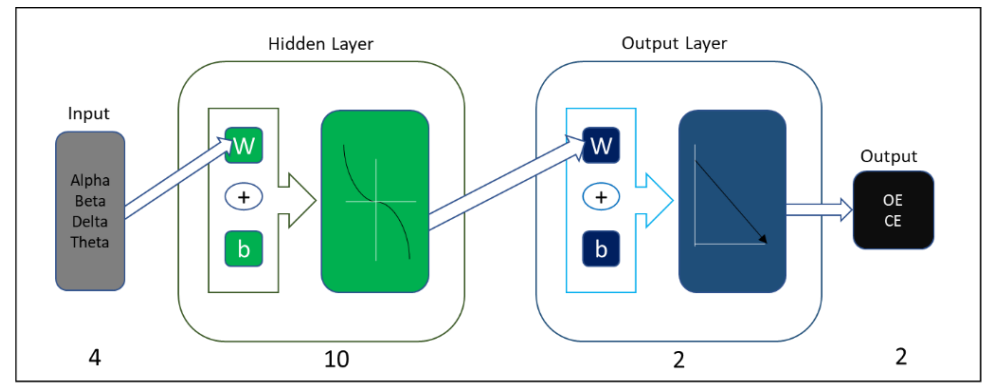

Figure 3. Artificial Neural Network model

\section{Results and Analysis}

The results captured from strokes patients' brain signals are addressed in this section. The original brain signal output is in time-domain. The data comprises alpha, beta, delta, and theta plus artefacts from the power line disturbance, baseline interference, and eye blinking contamination. The Power Spectrum Density (PSD) of the EEG signal was extracted after translating it to the timefrequency domain using FFT.

Figure 4 displays the features of each sub-band data plot for both open eyes and close eye conditions. From the displayed data plot, the range of power of each level is inconsistent. Various behavior and patterns of EEG sub-bands are noticeable. This indicates the variability of patterns for each of the brainwave sub-bands in this brainwave activity in each of the subject. The additional brain signal processing approach thus promotes a deeper understanding of the EEG mechanism as well as an analysis of its physiological importance.

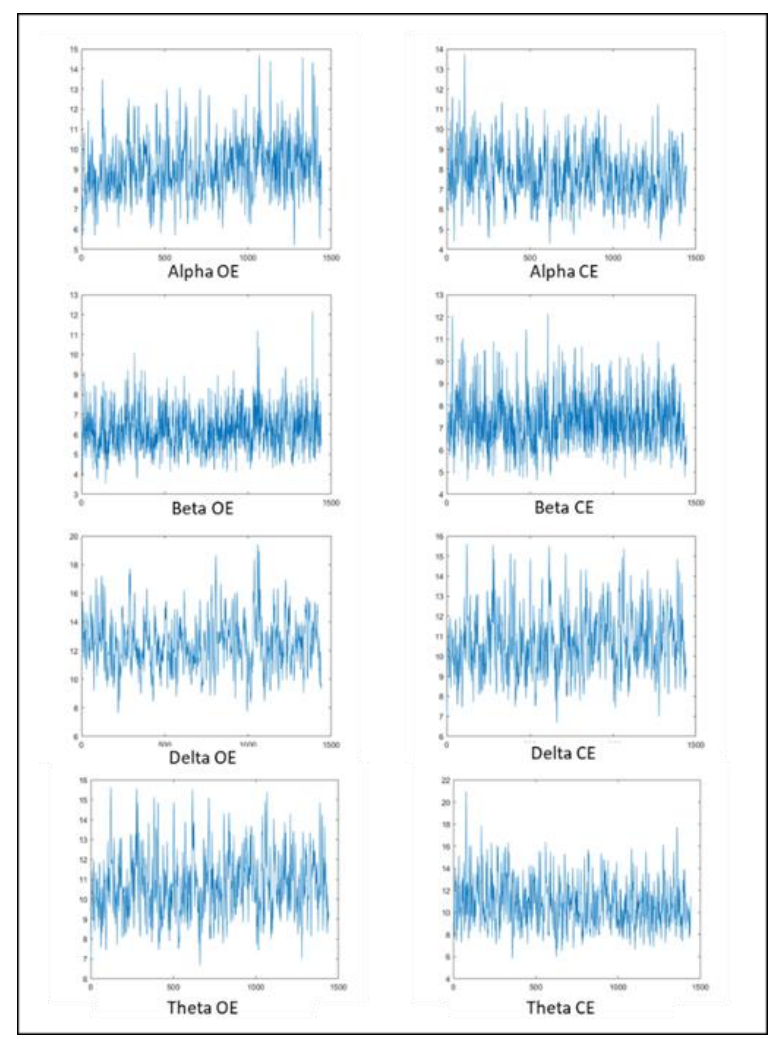

Figure 4. Alpha, Beta, Delta and Theta band power data plot of 14 subjects

\subsection{Neural Network Model during the Open-Close Eyes Task}

MATLAB R2017b software had been used to classify the collected data. The network architecture 
optimized for the structure comprises 4 input, 10 hidden, and 2 output nodes. Table 2 presents a summary of classification performance. Subject 2 provides the highest classification accuracy while subject 5 has the lowest classification accuracy. Subject 2 yields $87.3 \%, 88.2 \%$, and $84.7 \%$ accuracy value for training, validation, and testing respectively while Subject 5 yields $59.9 \%, 58.6 \%$, and $56.7 \%$ accuracy value for training, validation, and testing respectively.

Table 1. Classification performance of stroke subjects

\begin{tabular}{|c|c|c|c|}
\hline \multirow{2}{*}{ Subject } & \multicolumn{3}{|c|}{ Performance Parameters } \\
\cline { 2 - 4 } & \multicolumn{3}{|c|}{ Accuracy } \\
\cline { 2 - 4 } & Training & Validation & Testing \\
\hline 1 & 80.0 & 81.7 & 80.3 \\
\hline 2 & 87.3 & 88.2 & 84.7 \\
\hline 3 & 71.8 & 73.4 & 70.6 \\
\hline 4 & 71.0 & 67.4 & 56.0 \\
\hline 5 & 59.9 & 58.6 & 58.6 \\
\hline 6 & 60.1 & 61.8 & 68.3 \\
\hline 7 & 69.9 & 67.1 & 82.4 \\
\hline 8 & 84.5 & 83.8 & 81.0 \\
\hline 9 & 80.3 & 82.4 & 84.0 \\
\hline 10 & 81.5 & 82.4 & 76.6 \\
\hline 11 & 69.2 & 66.7 & 78.2 \\
\hline 12 & 73.9 & 69.5 & 82.2 \\
\hline 13 & 77.8 & 78.2 & 82.9 \\
\hline 14 & 83.8 & \multicolumn{2}{c|}{} \\
\hline
\end{tabular}

The data obtained are plotted in Figure 5 with a supplemental visual representation for a better understanding. All the values shown in the graph are actual results. The blue bar refers to the results corresponding to the training data. The orange bar refers to the results corresponding to the validation data while the grey bar refers to the testing data. It can be observed clearly that subjects 2 provide the highest accuracy value while subject 5 provides the lowest accuracy value.

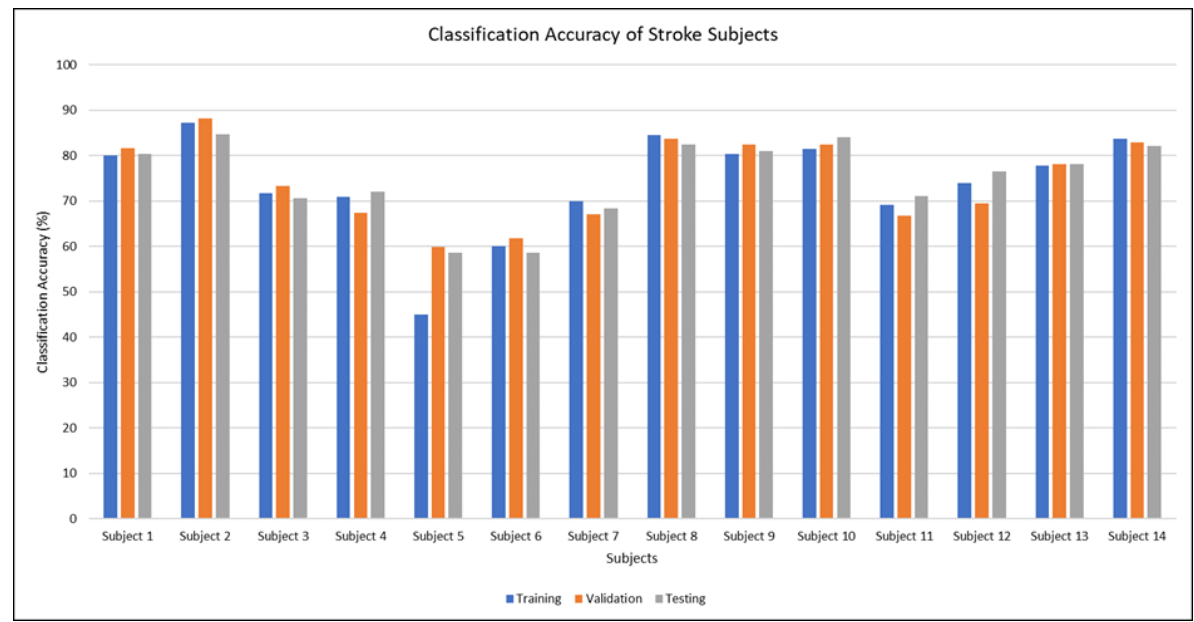

Figure 5. Classification Accuracy of Stroke Subjects

\section{Conclusion}

The ANN classifier successfully described the behavioral patterns of the various brainwave signal states for 14 patients suffering from a stroke. This method can recognize and classify 2 activities that validate our collection of data Improvements must be made to avoid misclassification in real-time. Our future work will consist, first of all, of gathering more data for other forms of tasks and thereby helping us to proceed with more interesting findings. We will then analyse and suggest another method of classification algorithm. another type of classification algorithm that can help to improve the classification performance by providing a greater accuracy value.

\section{Acknowledgment}

The research is financially sponsored by UTHM for TIER 1 Grant (H273) and GPPS (H407). The authors would like to express their appreciation to the representatives of NASAM Staff and stroke 
patients who agreed to cooperate in the data collection process and Multi-Disciplinary Research Group (MDRG) FKEE, UTHM for their encouragements.

\section{References}

[1] S. M. Hatem, G. Saussez, M, V. Prist, X. Zhang, D. Dispa and Y. Bleyenheuft, "Rehabilitation of Motor Function after Stroke: A Multiple Systematic Review Focused on Techniques to Stimulate Upper Extremity Recovery", Frontiers in Human Neuroscience, vol. 10, pp. 1-36, 2016, doi:10.3389/fnhum.2016.00442.

[2] K. Chenane and Y. Touati, "EEG Signal Classification for BCI based on Neural Network", 2018 IEEE International Conference on Bioinformatics and Biomedicine (BIBM) EEG, pp. 2573-2576, 2018.

[3] R. Mohanty, A. M. Sinha, A.B. Remsik, K.C. Dodd, BM. Young, T. Jacobson, M. McMillan, J. Thoma, H. Advani, V. A. Nair, T.J. Kang, K. Caldera, D. F. Edwards, J. C. Williams and Vivek Prabhakaran, "Machine learning classification to identify the stage of brain-computer interface therapy for stroke rehabilitation using functional connectivity", Frontiers in Neuroscience, vol. 12, no. MAY, pp. 1-14, 2018, doi:10.3389/fnins.2018.00353.

[4] D. C. Irimia, R. Ortner, M. S. Poboroniuc, B. E. Ignat, and C. Guger, “High Classification Accuracy of a Motor Imagery Based Brain-Computer Interface for Stroke Rehabilitation Training", Frontiers in Robotics and AI, vol. 5, no. November, pp. 1-9, 2018, doi:10.3389/frobt.2018.00130.

[5] A. A. Qureshi and A. Elmeligi, "Ischemic Stroke Detection using EEG Signals", Proceedings of CASCON'18, Markham, Ontario Canada, 2018.

[6] F. Lotte, "A Tutorial on EEG Signal Processing Techniques for Mental State Recognition in Brain-Computer Interfaces", Guide to Brain-Computer Music Interfacing, Springer, 2014, doi.org/10.1007/978-1-4471-65842 -7.

[7] C. M. Mccrimmon, C. E. King, P. T. Wang, S. C. Cramer, Z. Nenadic, and A. H. Do, “Brain-controlled functional electrical stimulation therapy for gait rehabilitation after stroke: a safety study", Journal of NeuroEngineering and Rehabilitation, 2015, doi:10.1186/s12984-015-0050-4.

[8] A. Al-nafjan, M. Hosny, Y. Al-Ohali, and A. Al-Wabil, "Review and Classification of Emotion Recognition Based on EEG Brain-Computer Interface System Research : A Systematic Review", Applied Science 2017, 2017, doi:10.3390/app7121239.

[9] A. B. M. A. Hossain, M. W. Rahman, and M. A. Riheen, "Left and right hand movements EEG signals classification using wavelet transform and probabilistic neural network", International Journal of Electrical and Computer Engineering, vol. 5, no. 1, pp. 92-101, 2015, doi: 10.11591/ijece.v5i1.pp92-101.

[10] D. B. Thiyam and E. R. Rajkumar, "A comparative performance analysis for classification of multiclass motor imagery movements", International Journal of Control Theory and Applications, vol. 9, no. 36, pp. 443-450, 2016.

[11] Y. Ma, X. Ding, Q. She, Z. Luo, T. Potter, and Y. Zhang, "Classification of Motor Imagery EEG Signals with Support Vector Machines and Particle Swarm Optimization", Computational and Mathematical Methods in Medicine, vol. 2016, pp. 1-8, 2016, doi:10.1155/2016/4941235.

[12] S. M. R. Islam, A. Sajol, X. Huang, and K. L. Ou, "Feature extraction and classification of EEG signal for different brain control machine", 2016 3rd International Conference on Electrical Engineering and Information and Communication Technology, iCEEiCT 2016, 2017, doi: 10.1109/CEEICT.2016.7873150.

[13] A. Suwannarat, S. Pan-ngum, and P. Israsena, "Comparison of EEG measurement of upper limb movement in motor imagery training system", BioMedical Engineering Online, vol. 17, no. 1, pp. 1-22, 2018, doi:10.1186/s12938-018-0534-0.

[14] A. N. Niranjani and M. Sivachitra, "Motor imagery signal classification using spiking neural network", Proceedings of the International Conference on Intelligent Sustainable Systems (ICISS), Palladam, 2017, pp. 901-904, doi: 10.1109/ISS1.2017.8389309.

[15] H. Cho, M. Ahn, and S. C. Jun, "A Step-by-Step Tutorial for a Motor Imagery-Based BCI", Brain-Computer Interfaces Handbook, pp. 445-446, March, 2018, doi:10.1201/9781351231954-23 .

[16] K. D. Morton, P. Torrione, L. Collins, and S. Keene, "An Open Source Pattern Recognition Toolbox for MATLAB", June, 2014, Available online at: http://newfolderconsulting.com/prt.Velliste.

(C) 2021 by the author(s). Published by Annals of Emerging Technologies in Computing (AETiC), under the terms and conditions of the Creative Commons Attribution (CC BY) license which can be accessed at http://creativecommons.org/licenses/by/4.0. 